\title{
Biosynthesis of Adipokinetic Hormones (AKHs): Further Characterization of Precursors and Identification of Novel Products of Processing
}

\author{
Siegfried Hekimi and Michael O'Shea \\ Laboratoire de Neurobiologie, $\mathrm{CH}-1211$ Genève 4, Switzerland
}

Two adipokinetic hormones (AKH I: pGlu-Leu-Asn-Phe-ThrPro-Asn-Trp-Gly-Thr-NH ${ }_{2}$, and AKH II: pGlu-Leu-Asn-Phe-SerThr-Gly-Trp- $\mathrm{NH}_{2}$ ) are synthesized by the neurosecretory cells of the corpora cardiaca (CC) of the locust Schistocerca gregaria. Both AKHs are released into the blood during flight and serve to regulate lipid metabolism and other physiological processes involved in flight. By in vitro "pulse-chase" experiments we show that 2 precursors (P1 and $\mathrm{P}_{2}$ ) are involved in AKH biosynthesis. These are about $8.4 \mathrm{kDa}$ polypeptides which are AKH immunoreactive but also contain an amino acid (tyrosine) not present in the AKH peptides. By following the fate of ${ }^{14} \mathrm{C}$-tyrosine incorporated into $\mathrm{P1}$ and P2, we have identified 2 novel products of precursor processing. These are called AKH-Precursor Related Peptides or APRP 1 and APRP 2. Size exclusion chromatography shows that the APRPs are about $6.5 \mathrm{kDa}$ large and therefore represent major fragments of the precursors.

The optical density peaks corresponding to P1, P2, APRP 1, and APRP 2 on a reverse-phase chromatogram are identified. The precursors being metabolic intermediates are represented by minor optical density peaks that disappear when de novo protein synthesis is blocked by cycloheximide. In contrast, the APRPs are represented by major optical density peaks consistent with their being accumulating end products of AKH precursor processing. The function of the APRPs is as yet unknown. They are, however, co-synthesized and also co-released with the AKHs, and may therefore also have hormonal functions related to flight.

It is now well established that the precursor for a peptide transmitter or hormone may include more than one bioactive amino acid sequence (for reviews, see Lynch and Snyder, 1986; Eipper et al., 1986). During enzymatic processing of the precursor, different bioactive sequences can therefore be liberated and made available for release. There are few intact tissue systems in which this aspect of peptide biosynthesis can conveniently be studied. One such system is represented by the bag cell cluster of Aplysia. These cells produce the precursor of egg laying hormone (ELH), which upon processing generates ELH and additional peptides that appear to be necessary for the expression of the complete egg laying behavior (Scheller et al., 1983; Mayeri et al., 1985;

\footnotetext{
Received May 2, 1988; revised Aug. 22, 1988; accepted Aug. 23, 1988.

This work was supported by Grant 3.181-0.85 from the Fond National Suisse

Correspondence should be addressed to Michael O'Shea, Cell Biology Laboratory, School of Life Sciences, New College (RHBNC), University of London, Egham, Surrey, England.

Copyright (C) 1989 Society for Neuroscience $0270-6474 / 89 / 030996-08 \$ 02.00 / 0$
}

Sigvardt et al., 1986). We have previously described (Hekimi and O'Shea, 1987) another tissue that allows us to study the biosynthesis and identify 2 precursors ( $\mathrm{P} 1$ and $\mathrm{P} 2)$ of 2 related insect neuropeptides (the adipokinetic hormones, or AKHs). Here, we identify 2 novel peptides generated from these precursors and characterize them as major fragments of their respective precursors.

Our studies were initiated with the adipokinetic hormones (AKH I and AKH II) synthesized by the neurosecretory cells of the glandular lobes of the corpora cardiaca (CC) of the locust Schistocerca gregaria. The CC are paired neurosecretory glands located in the head in close proximity to the brain, by which they are innervated. In the adult locust the $\mathrm{AKH}$ peptides are released just after the initiation of flight. This results in a mobilization of lipid metabolism, which provides the energy for migratory flight. The amino acid sequence of AKH I is pGluLeu-Asn-Phe-Thr-Pro-Asn-Trp-Gly-Thr- $\mathrm{NH}_{2}$ (Stone et al., 1976) and for AKH II pGlu-Leu-Asn-Phe-Ser-Thr-Gly-Trp- $\mathrm{NH}_{2}$ (Siegert et al., 1985). These hormones are major products of the neurosecretory cells of the locust CC. The CC (about $0.1 \mathrm{mg}$ wet weight) contain as much as $1 \mathrm{nmol} \mathrm{AKH} \mathrm{I}$ and $0.2 \mathrm{nmol}$ AKH II. This remarkable tissue therefore provides an unusually good system for the analysis of the synthesis of neuropeptides.

Synthesis of AKH I and AKH II can be studied by providing radiolabeled amino acids to $\mathrm{CC}$ maintained in short-term tissue culture (Hekimi and O'Shea, 1987). By following the incorporation of ${ }^{3} \mathrm{H}$-tryptophan, we showed that label is first incorporated in 2 polypeptides called $\mathrm{P} \mathbf{l}$ and $\mathrm{P} 2$ prior to its appearance in AKH I and II. On the basis of this observation and by showing that $\mathrm{P} 1$ and $\mathrm{P} 2$ are $\mathrm{AKH}$-immunorcactive, we identified them as precursors of the AKHs. In addition, we found that biosynthetically labeled P1 and P2 and their AKH immunoreactivity co-migrated with 2 major optical density (OD) peaks detected using reverse-phase chromatography. This led us to believe that the 2 OD peaks were due to the presence of relatively large amounts of the $\mathrm{AKH}$ precursors. In this respect, we were wrong. We show here that $\mathrm{P} 1$ and $\mathrm{P} 2$ and their AKH immunoreactivity can be separated from the major OD peaks. Relatively small optical density peaks are now assigned to the precursors, consistent with $\mathrm{P} 1$ and $\mathrm{P} 2$ being nonaccumulating intermediates of AKH biosynthesis. We show now that the large OD peaks are produced by 2 major fragments of the precursors, generated during processing. They accumulate in the $\mathrm{CC}$ in amounts comparable to the amounts of the AKHs. We have named these newly identified products $A$ dipokinetic hormone-Precursor $R \mathrm{e}$ lated Peptides, or APRPs. Although derived from the AKH precursors, the APRPs are themselves neither AKH-immuno- 


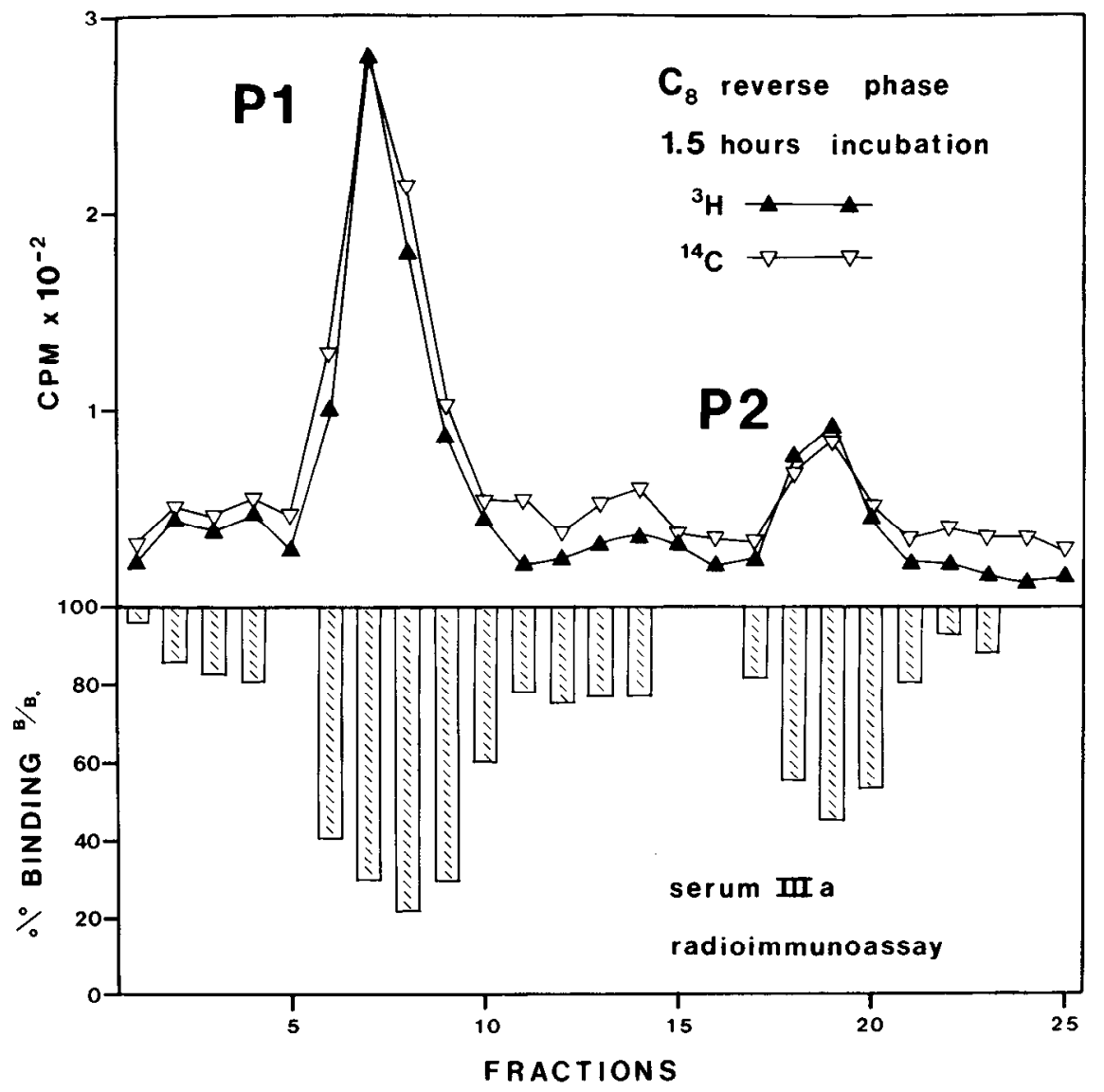

Figure 1. Reverse-phase chromatogram of $30 \mathrm{CC}$ showing double-labeled ( ${ }^{3} \mathrm{H}$-tryptophan and ${ }^{14} \mathrm{C}$-tyrosine) $\mathrm{P} 1$ and P2 (upper panel) and the AKH-like immunoreactivity associated with each fraction of the eluant measured by RIA (lower panel). Note that the peak fraction of the 2 isotopes coelutes for both $P 1$ and P2, and, moreover, the shapes of the peaks defined by the 2 isotopes are the same. The peaks of double-labeled precursors also coelute with the AKH-like immunoreactivity. In this experiment each fraction was split in 2 parts, one being subjected to RIA corresponding to $25 \mathrm{CC}$ and the other was monitored for ${ }^{3} \mathrm{H}$ - and ${ }^{14} \mathrm{C}$-decays. reactive nor do they contain tryptophan. While their function is unknown, their synthesis is coupled to that of the AKHs, and we show that they are released with them. The APRPs might therefore be hormones with activities that complement those of the AKHs (see Discussion).

\section{Materials and Methods}

Animals. Both male and female adult specimens of the locust Schistocerca gregaria were used in our experiments. They were reared in our own laboratory culture. Female New Zealand White Rabbits produced the antiscrum to a synthetic analog of $\triangle \mathrm{KH} \mathrm{I}$.

Preparation of $A K H$ antiserum and radioimmunoassay procedures. To generate an antiserum to the precursor form of $\mathrm{AKH}$, an analog of the sequence Lys-Tyr-Leu-Asn-Phe-Thr-Pro-Asn-Trp-Gly-Thr-OH was designed and synthesized (Peninsula Laboratories). Details of methods used to generate antisera and those used in the radioimmunoassay (RIA) were given in Hekimi and O'Shea (1987), in which the serum is named IIIa. Here, the tracer for the radioimmunoassay (RIA) was a synthetic analog of the sequence Tyr-Leu-Asn-Phe-Thr-Pro-Asn-Trp-Gly-Thr$\mathrm{NH}_{2}$ (Peninsula Laboratories). This peptide was radiolabeled on the tyrosine residue with ${ }^{125} \mathrm{I}$. Activated charcoal was used to precipitate unbound peptide after serum IIIa (diluted 20 -fold) was incubated with the tracer and samples. The ability of material from HPLC fractions to displace bound counts was determined.

Organ (CC) culture. Details of in vitro incubation methods were given in Hekimi and O'Shea (1987). In order to monitor synthesis of the precursors and their products $10 \mu \mathrm{Ci}$ of ${ }^{3} \mathrm{H}$ [side chain 2,3] L-tryptophan $(60 \mathrm{Ci} / \mathrm{mm})$ or $10 \mu \mathrm{Ci}$ of $\mathrm{L}-\left(\mathrm{U}-{ }^{14} \mathrm{C}\right)$ tyrosine $(500 \mathrm{mCi} / \mathrm{mm})$ or both was added to the incubation saline. Following varying durations of incubation (see Results) with labeled amino acids, the CC were either washed in saline and extracted (see below) or transferred to a large volume ( 5 $\mathrm{ml}$ ) of "translation-block" medium. The translation-block medium consisted of saline containing $1 \mu \mathrm{g} / \mathrm{ml}$ cycloheximide.

Extraction, identification, and purification procedures. Corpora cardiaca were homogenized in $100 \mu \mathrm{l}$ of $0.1 \%$ trifluoroacetic acid (TFA) at room temperature with a Branson microprobe ultrasound cell disruptor. The crude TFA-soluble supernatant was removed, frozen immediately, and subjected to size-exclusion and or reverse-phase chromatography.

For size fractionation, a $60 \mathrm{~cm}$ TSK $2000 \mathrm{SW}$ gel-permeation column (LKB), eluted at $0.5 \mathrm{ml} / \mathrm{min}$ with $0.1 \%$ TFA containing $15 \%$ (vol/vol) acetonitrile was used. The exclusion size of this column is about 50 $\mathrm{kDa}$. Acetonitrile was used to prevent hydrophobic interactions, which can result in anomalous retentions of some peptides and tryptophan The TSK 2000 column was calibrated according to molecular weight using the following standards: myoglobin (fragment) $14.4 \mathrm{kDa}$; myoglobin (fragment) $8.2 \mathrm{kDa}$; myoglobin (fragment) $6.2 \mathrm{kDa}$; a synthetic 28 residue peptide of $3.2 \mathrm{kDa}$. Reverse-phase separations of AKH I, AKH II, APRP 1, APRP 2, P1, and P2 were achieved using a $\mathrm{C}_{8}$ widepore column (Aquapore RP-300; $7 \mu \mathrm{m}$, Pierce Chemical Company). The column was eluted at $1.5 \mathrm{ml} / \mathrm{min}$ with $0.1 \%$ TFA and a linear acetonitrile gradient (from 25 to $45 \%$ in $10 \mathrm{~min}$ ). Retention times of synthetic AKH I or II (Peninsula Laboratories) could not be distinguished from those of native unlabeled and native biosynthetically labeled AKH I or II.

Three types of detection systems were used to identify the eluting compounds. On-line detection was provided by a Waters 440 fixedwavelength absorbance detector set at $214 \mathrm{~nm}$, which gave an estimate of the relative abundance of peptide bonds. In one experiment (Fig. 1), compounds were detected by RIA and scintillation counting after fractionation of the eluant. For detection of the AKH immunoreactivity, HPLC fractions were evaporated to dryness using a Savant Speed-Vac evaporator and subjected to RIA (see above). For calculation of the isotopic content of HPLC fractions, ${ }^{3} \mathrm{H}$ and ${ }^{14} \mathrm{C} \beta$-emissions were distinguished by their respective cncrgics.

Potassium-stimulated calcium-dependent release. The ability of $\mathrm{CC}$ to release the APRPs was investigated by superfusing the glands with physiological saline and with salines of altered ionic composition. In order to concentrate released hydrophobic compounds, the glands were placed in a small chamber (Swinny Filter Holder, Millipore) attached above a cartridge containing reverse-phase packing $\left(\mathrm{C}_{18}\right.$ SepPak, Waters Associate). The superfusate was added above the chamber, and a peri- 

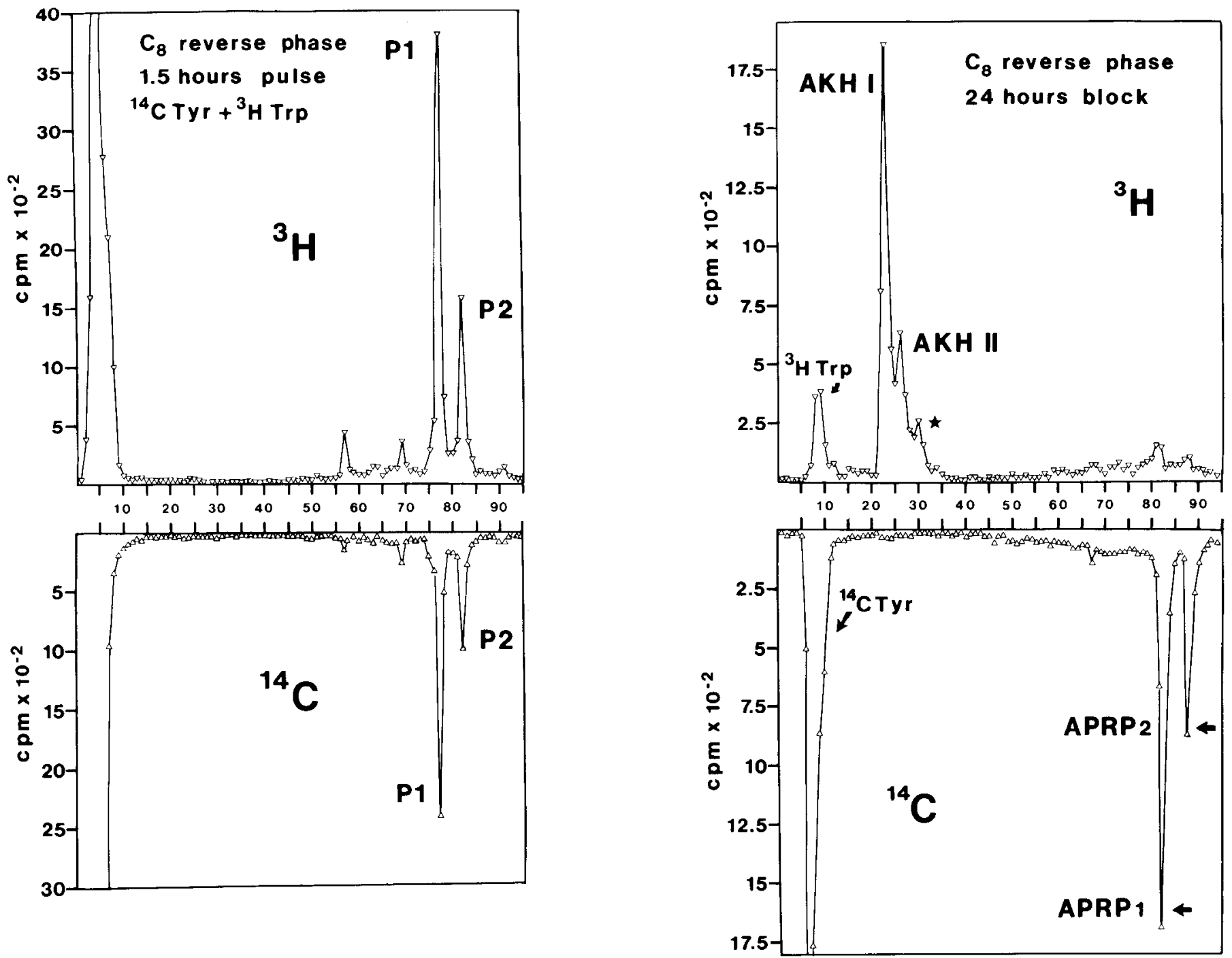

Figure 2. Pulse-translation block experiment using reverse-phase chromatography of $\mathrm{CC}$ after incubation with ${ }^{14} \mathrm{C}$-tyrosine and ${ }^{3} \mathrm{H}$-tryptophan for $1.5 \mathrm{hr}$ followed by $24 \mathrm{hr}$ block. Left panel, Chromatogram just after the incubation period. Both isotopes are incorporated into both P1 and $\mathrm{P} 2$ as indicated by the co-migration of the ${ }^{3} \mathrm{H}$ (above) and ${ }^{14} \mathrm{C}($ below). Right panel. Chromatogram of CC incubated in cycloheximide for 24 hr after the $1.5 \mathrm{hr}$ pulse labeling. Label $\mathrm{P} 1$ and $\mathrm{P} 2$ is now transferred to the AKHs $\left({ }^{3} \mathrm{H}\right)$ and the APRPs $\left({ }^{14} \mathrm{C}\right)$.

staltic pump was used to move it through the chamber and SepPak. Further details of this method for monitoring peptide release have been described (Adams and O'Shea, 1983; O'Shea et al., 1984). Fifty CC were placed in the chamber and superfused for $1 \mathrm{hr}$ each in succession with 3 types of saline. The first was physiological saline, the second contained no calcium but had elevated potassium concentration (50 $\mathrm{mm}$ ), and the third had elevated potassium concentration with normal $(5 \mathrm{~mm})$ calcium. For each saline condition a new activated SepPak was attached to the chamber. The SepPaks were flushed with distilled water and the trapped peptides eluted in $5 \mathrm{ml}$ of methanol. Following evaporation of the methanol, the samples were analyzed by reverse-phase chromatography for the presence of APRPs.

\section{Results}

Identification of 2 AKH-precursor related peptides (APRPS)

In vitro incubation of $\mathrm{CC}$ with ${ }^{3} \mathrm{H}$-tryptophan results in the synthesis of 4 major tryptophan-containing peptides. These are AKH I, AKH II, and their precursors P1 and P2 (Hekimi and O'Shea, 1987). In our previous publication, the AKH-precursor status of P1 and P2 was established by the following criteria. Both $\mathrm{P} 1$ and $\mathrm{P} 2$ are larger than the $\mathrm{AKH}$ peptides and both are synthesized prior to them. By performing pulse-chase or pulse- translation block experiments, tryptophan incorporated in P1 and P2 can be recovered in AKH I and II. Finally, both P1 and $\mathrm{P} 2$ are $\mathrm{AKH}$-immunoreactive, indicating that they contain amino acid sequences related to AKH.

In order to investigate whether $\mathrm{P} 1$ and $\mathrm{P} 2$ could contain peptides other than $\mathrm{AKH}$, we cultured $\mathrm{CC}$ in the presence of ${ }^{3} \mathrm{H}$ tryptophan, an amino acid present in the AKHs and ${ }^{14} \mathrm{C}$-tyrosine, an amino acid not present in the AKHs. Figure 1 shows a $\mathrm{C}_{8}$ reverse-phase chromatogram of an extract of $\mathrm{CC}$ that was incubated for $1.5 \mathrm{hr}$ with both ${ }^{3} \mathrm{H}$-tryptophan and ${ }^{14} \mathrm{C}$-tyrosine. Two peaks labeled with both isotopes $\left({ }^{3} \mathrm{H}\right.$ and $\left.{ }^{14} \mathrm{C}\right)$ can be seen. These were identified as $\mathrm{P} 1$ and $\mathrm{P} 2$ by several criteria. For example, they occur with the expected retention time and amplitudes (the peak of $\mathrm{P} 1$ predominates) and both are AKHimmunoreactive (see lower part of Fig. 1). Note that the ${ }^{3} \mathrm{H}$ and ${ }^{14} \mathrm{C}$ peaks are virtually superposed, indicating that tyrosine, in addition to tryptophan (as shown previously), is incorporated in the AKH precursors. Thus, the processing of $\mathrm{P} 1$ and $\mathrm{P} 2 \mathrm{might}$ result in the production of tyrosine-containing peptides, in addition to the AKHs. 


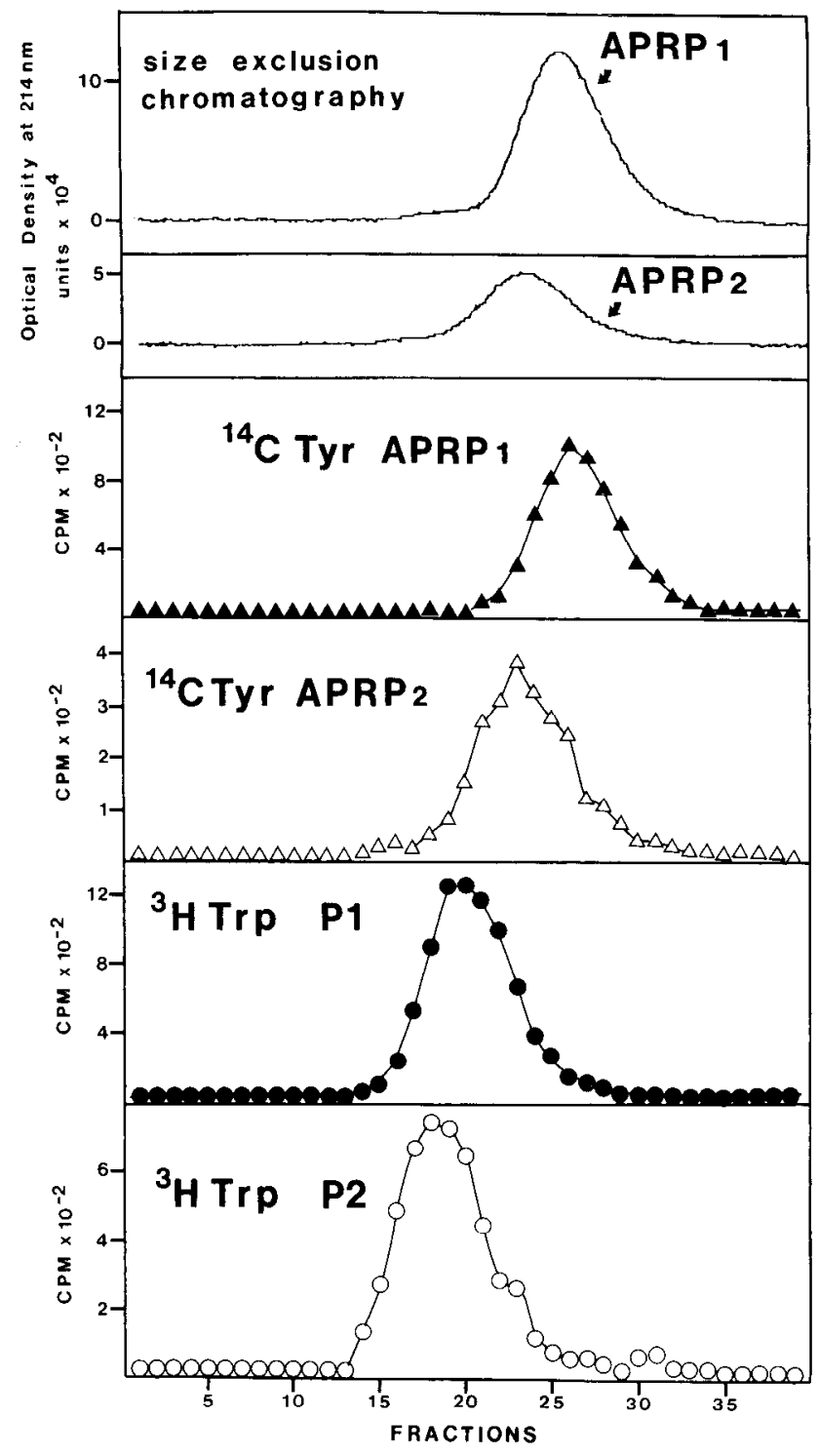

Figure 3. Size characterization by TSK 2000 size exclusion chromatography of reverse-phase purified P1, P2, APRP 1, and APRP 2. The upper panel shows the OD at $214 \mathrm{~nm}$ associated with the chromatographic profiles of the labeled compounds. Two chromatographic runs were performed, one included purified Pl plus APRP 1 and the other P2 plus APRP 2 (equal CC equivalents for each). Note that the OD peaks co-migrate with the peaks of labeled APRPs and not with the labeled Ps.

That this is the case is shown in Figure 2, in which CC pulselabeled with ${ }^{3} \mathrm{H}$-tryptophan and ${ }^{14} \mathrm{C}$-tyrosine are placed in a "translation-block" medium containing cycloheximide. Note that during the brief incubation ( $1.5 \mathrm{hr}$ ) no radiolabeled $\mathrm{AKH}$ is found. Following a $24 \mathrm{hr}$ period of translation block, the doublc-labcled precursors have disappeared and the tritium tryptophan is chased into the $2 \mathrm{AKH}$ peptides, as expected. Transfer of ${ }^{3} \mathrm{H}$-tryptophan to the $2 \mathrm{AKHs}$ is accompanied by the transfer of ${ }^{14} \mathrm{C}$-tyrosine into 2 new radiolabeled compounds (APRP 1 and 2), migrating close to the double-labeled precursors (now absent).

The fact that APRP 1 and 2 migrate on a reverse-phase column very close to the $\mathrm{P} 1$ and $\mathrm{P} 2$ precursors suggests they are similar in hydrophobicity to their parent compounds. This could
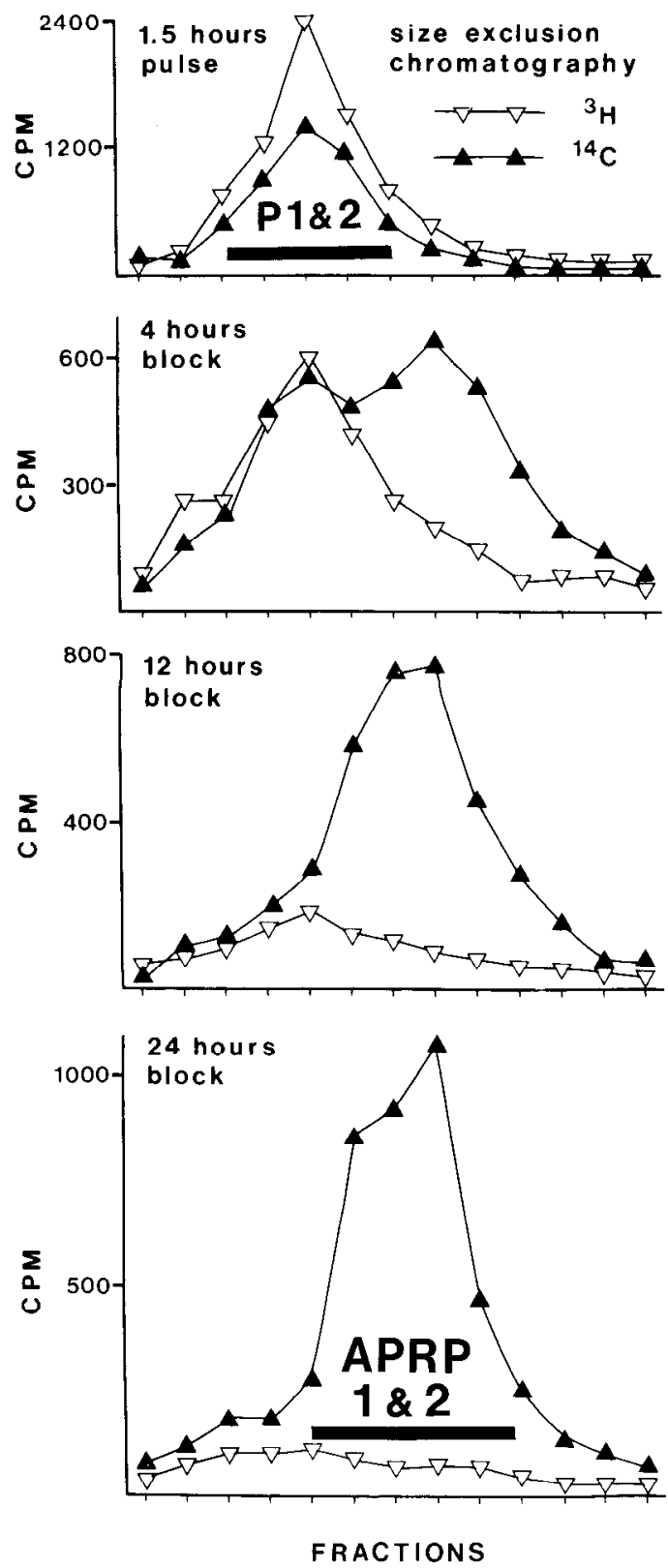

Figure 4. Pulse-translation block time-course experiment showing sizeexclusion chromatograms of $\mathrm{CC}$ after incubation for $1.5 \mathrm{hr}$ with ${ }^{3} \mathrm{H}-$ tryptophan and ${ }^{14} \mathrm{C}$-tyrosine. Immediately following the incubation $\mathrm{CC}$ were extracted and chromatographed (top) and then after 4, 12, and 24 $\mathrm{hr}$ in the presence of cycloheximide. The figure shows a progressive transfer of ${ }^{14} \mathrm{C}$-tyrosine from the larger Ps to the smaller APRPs during translation block. As the double-labeled peak (P1 and P2) disappears the ${ }^{3} \mathrm{H}$-labeled tryptophan in this peak is transferred to the $\mathrm{AKH}$ peptide (not included in these chromatograms) and the ${ }^{14} \mathrm{C}$-tyrosine is transferred to the APRPs. Thus, the ${ }^{14} \mathrm{C}$-tyrosine is shifted progressively to the right (smaller molecular weight).

arise if they represent major fragments of the precursors. Sizing of the APRPs confirms this. To investigate the size relationships between the newly identified APRPs and their precursors we first purified P1, P2, and APRP 1 and 2 by reverse-phase chromatography and then chromatographed them by size exclusion. This is shown in Figure 3. In this experiment CC were pulse labeled for $1.5 \mathrm{hr}$ with either ${ }^{3} \mathrm{H}$-tryptophan or ${ }^{14} \mathrm{C}$-tyrosine. The tryptophan-labeled $\mathrm{P} 1$ and $\mathrm{P} 2$ were separated and purified by $\mathrm{C}_{8}$ reverse-phase HPLC. The glands pulsed with ${ }^{14} \mathrm{C}$-tyrosine, 


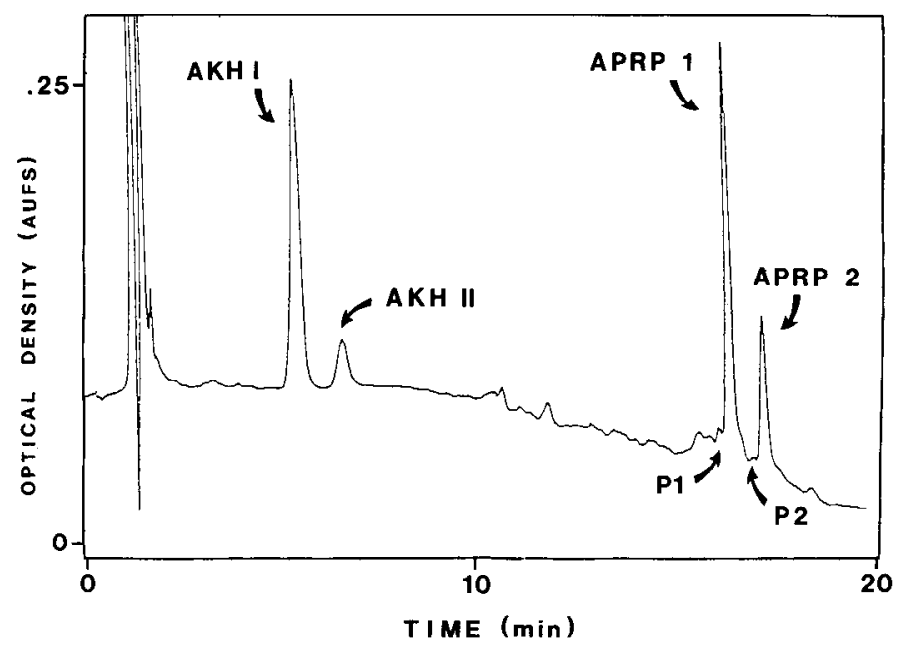

Figure 5. Reverse-phase chromatogram of 3 adult CC (glandular lobes). Only 4 major optical density $(214 \mathrm{~nm})$ peaks are seen, AKH I and II and APRP 1 and APRP 2. The first peaks seen to the left represent the solvent front. The simplicity of the OD profile suggests that the 4 peaks seen represent the major stored products of the neurosecretory cells of the glandular lobe.

however, were transferred to the translation-block medium overnight to allow processing of the pulse-labeled P1 and P2 into the ${ }^{14} \mathrm{C}$-labeled APRPs. These glands were also subjected to $\mathrm{C}_{8}$ reverse-phase chromatography and the $2{ }^{14} \mathrm{C}$-labeled products (APRP 1 and 2) collected separately. Thus, we produced purified ${ }^{3} \mathrm{H}$-tryptophan $\mathrm{P} 1$ and $\mathrm{P} 2$ and purified ${ }^{14} \mathrm{C}$-tyrosine APRP 1 and APRP 2.

These purified compounds were then applied to a size-exclusion column (TSK 2000) in the following way (Fig. 3). The ${ }^{3} \mathrm{H}-$ labeled P1 was mixed with ${ }^{14} \mathrm{C}$-labeled APRP 1 and similarly labeled P2 was mixed with labeled APRP 2. The mixtures included the same tissue (CC) equivalent amounts of the 2 compounds ( $\mathrm{P}$ and an APRP). This allowed us to see the relative molecular weights of a $P$ and an APRP simultaneously while monitoring the relative contribution made by each to the OD (a measure of relative amounts per gland). Three expectations were confirmed by this experiment. First, the APRPs are smaller than the Ps. Second, the $\Lambda$ PRPs are only slightly smaller than the Ps, indicating that they are major fragments of their precursors. Third, monitoring of the column eluant at $214 \mathrm{~nm}$ shows that the APRPs are present in the $\mathrm{CC}$ in much larger amounts than the Ps (see also Fig. 6). This is expected because precursors are metabolic intermediates but their products accumulate.

Estimates of sizes were derived from the TSK 2000 column calibrated using polypeptide standards spanning the range from 14.4 to $3.2 \mathrm{kDa}$ (see Materials and Methods). This gave molecular weights of about $8.4 \mathrm{kDa}$ for $\mathrm{P} 1$ and $\mathrm{P} 2$ and about 6.5 $\mathrm{kDa}$ for APRP 1 and APRP 2. Thus, the APRPs represent approximately $80 \%$ of the size of their precursors. The difference in size (about $2 \mathrm{kDa}$ ) between a $P$ and an APRP allows the precursors to include no more than 2 copies of an AKH peptide (see Discussion).

The separation of the Ps from the APRPs achieved by the TSK 2000 column allowed us to follow the time dependence of the transfer of ${ }^{14} \mathrm{C}$-tyrosine from the larger Ps into the smaller APRPs. We exploited the fact that Ps can be double-labeled (with ${ }^{14} \mathrm{C}$-tyrosine and ${ }^{3} \mathrm{H}$-tryptophan) and that the tyrosine is transferred to the APRPs after processing. In this way we have been able to show the expected time-dependent transfer of label from precursors $(\mathrm{P} 1+\mathrm{P} 2)$ into the APRPs. This experiment is shown in Figure 4. The CC were first incubated for $1.5 \mathrm{hr}$ in ${ }^{3} \mathrm{H}$-tryptophan and ${ }^{14} \mathrm{C}$-tyrosine, thus labeling $\mathrm{P} 1$ and $\mathrm{P} 2$. Immediately following this incubation, a broad double-labeled peak representing the sum of $\mathrm{P} 1$ and $\mathrm{P} 2$ is seen by size-exclusion chromatography (Fig. 4, upper panel). That this broad peak consists of $\mathrm{P} 1$ and $\mathrm{P} 2$ can be confirmed by collecting this material and rechromatographing it by $\mathrm{C}_{8}$ reverse phase. Following the pulse labeling, $\mathrm{CC}$ were transferred into the translation-block medium and then sampled periodically. Figure 4 shows the results following periods of 4,12 , and $24 \mathrm{hr}$ in cycloheximide. Since during these periods new translation but not processing is blocked, there is a progressive, time-dependent decline in the double-labeled $\mathrm{P} 1+\mathrm{P} 2$ peak. The ${ }^{3} \mathrm{H}$-tryptophan is transferred to the AKH I and II peptides (Hekimi and O'Shea, 1987; not shown in Fig. 4). The ${ }^{14} \mathrm{C}$-tyrosine of the double-labeled peak is shifted slightly to the right, as tyrosine-labeled APRPs are synthesized. When chromatographed together, APRP 1 is not well resolved from APRP 2, resulting in the bilobed appearance of the APRP peak at the end of the $24 \mathrm{hr}$ period in cycloheximide (Fig. 4, lower panel).

\section{$O D$ peaks assigned to $P$ s and APRPs}

The results so far can be summarized by saying that 2 precursors P1 and P2 (about $8.4 \mathrm{kDa}$ ) are synthesized and metabolized and processed rapidly in the $\mathrm{CC}$, producing 4 accumulating compounds, AKH I and II (1.1 and $0.8 \mathrm{kDa})$ and APRP 1 and 2 (each about $6.5 \mathrm{kDa}$ ). The APRPs therefore represent large fragments of the Ps, the difference in size being about 1 or 2 AKH peptides. Size considerations suggest that the AKHs and APRPs may be the only accumulating products of AKH-precursor processing in the CC. A reverse-phase chromatogram of a $\mathrm{CC}$ extract reflects this simplicity (Fig. 5) in which 4 major optical density peaks are seen. In Figure 5, the optical density peaks marked APRP 1 and APRP 2 co-migrate with the biosynthetically labeled products of $\mathrm{P} 1$ and $\mathrm{P} 2$ processing (see Fig. 2). Note that each APRP is preceded by a very minor optical density peak, barely visible in Figure 5 but more clearly shown in Figure 6. These peaks were not detected in our previous report (Hekimi and O'Shea, 1987) because the $\mathrm{C}_{8}$ column (Watman Protesil $300 \AA$ octyl) and conditions used in those experiments did not permit the separation of the smaller from the larger OD peaks. The chromatogram shown in Figure 6 is from a Pearce $\mathrm{C}_{8}$ column using a gradient designed to optimize separation in this region (see Materials and Methods). We can now show that the ${ }^{3} \mathrm{H}$-tryptophan-labeled $\mathrm{P} 1$ and $\mathrm{P} 2$ do not co-migrate with Imajor OD peaks but with the 2 minor OD peaks. This was to be expected from the results shown in Figure 3 in which significant $O D$ is associated with the APRPs and not the Ps.

If the 2 minor OD peaks co-migrating with biosynthetically labeled precursors are indeed due to the precursors, their amplitudes should be diminished when $\mathrm{P} 1$ and $\mathrm{P} 2$ are processed under conditions that prevent their continued synthesis. Such conditions are achieved by placing the glands in cycloheximide. So we performed the following experiment. Corpora cardiaca were first incubated in the presence of both ${ }^{14} \mathrm{C}$-tyrosinc and ${ }^{3} \mathrm{H}$ tryptophan for $1.5 \mathrm{hr}$. During this brief period the P1 and P2 precursors become double-labeled, as shown previously. The biosynthetically labeled P1 and P2 co-migrate with the 2 minor OD peaks (Fig. 6, left panel). After pulse labeling some of the 

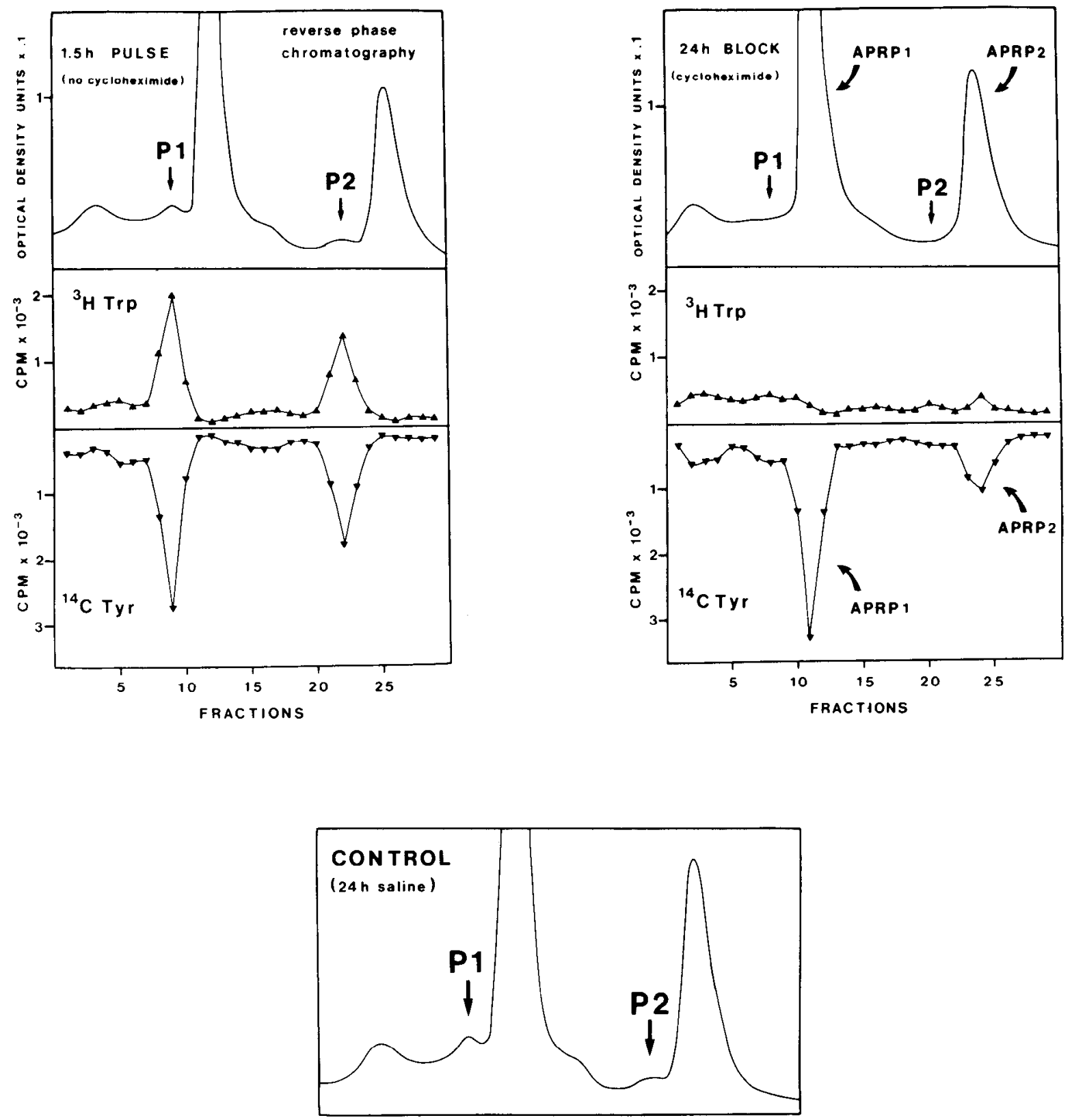

Figure 6. A pulse-translation block experiment using reverse-phase chromatography. In the left panel, $\mathrm{P} 1$ and $\mathrm{P} 2$ were double-labeled with ${ }^{3} \mathrm{H}-$ tryptophan and ${ }^{14} \mathrm{C}$-tyrosine for a $1.5 \mathrm{hr}$ pulse incubation. Note that the double-labeled precursors do not co-migrate with the major OD peaks but with the minor ones shown above. After $24 \mathrm{hr}$ in cycloheximide (right panel), the double-labeled precursors have disappeared and so have the small OD peaks co-migrating with them. The ${ }^{14} \mathrm{C}$-tyrosine peaks are now shifted to the right and each lies under a major OD peak. The lower panel shows that the small OD peaks associated with the PI and P2 precursors are not diminished after $24 \mathrm{hr}$ in physiological saline without cycloheximide. Note in right panel that only the P1 and P2 associated OD peaks are lost, the third minor peak eluting earlier being unchanged in all panels.

$\mathrm{CC}$ were transferred to the translation-block medium for $24 \mathrm{hr}$. Following this, ${ }^{14} \mathrm{C}$-labeled tyrosine no longer co-migrates with $\mathrm{P} 1$ and $\mathrm{P} 2$ but is shifted to the right to lie under the larger OD peaks produced by the APRPs (Fig. 6, right panel). This result was expected from previous experiments (Fig. 2). Note that the ${ }^{3} \mathrm{H}$-tryptophan is no longer present because it has been transferred to the AKHs (not shown in Fig. 6). The new finding shown here is that the disappearance of label from P1 and P2, indicating complete processing of available precursor pool, is accompanied by the disappearance of the small OD peaks. Thus, in conditions under which new precursor synthesis is prevented, the small OD peaks behave like the biosynthetically labeled precursor peaks, that is, they are "chased out." In a control experiment, some of the glands that received the double-labeled pulse were washed and transferred to saline without cycloheximide for 24 hr. Under these conditions, we would not expect the steadystate levels of the precursors to change because P1 and P2 synthesis can continue. The OD associated with $\mathrm{P} 1$ and $\mathrm{P} 2$ therefore should not be diminished. This is confirmed in the lower panel of Figure 6.

\section{Calcium-dependent release of the APRPS}

The demonstration of co-synthesis of the APRPs and the AKH peptides suggests that the APRPs may be hormones. Questions 


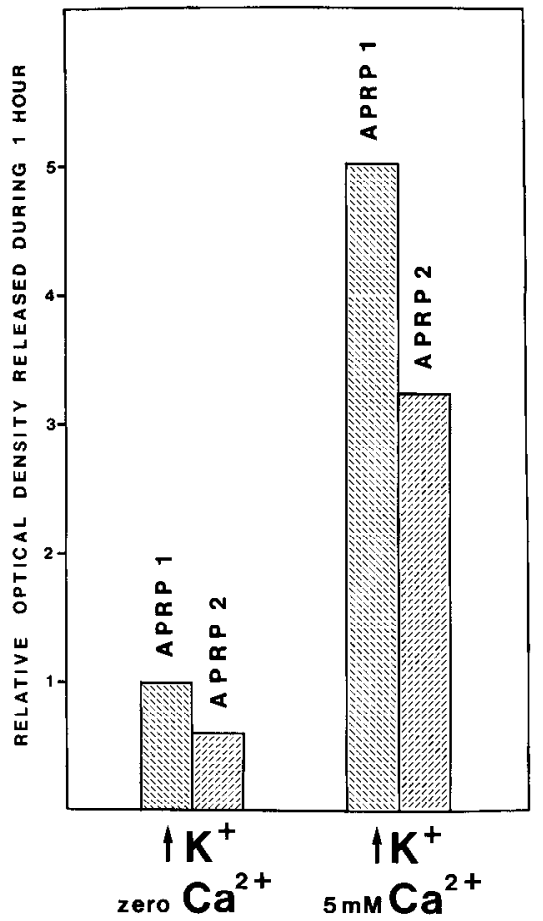

Figure 7. Calcium-dependent release of APRP 1 and APRP 2 from the CC activated by high $(50 \mathrm{~mm})$ potassium saline. The released peptides were analyzed by reverse-phase chromatography and comparison of release made by measuring optical density at $214 \mathrm{~nm}$ (relative scale: the OD associated with APRP 1 released under zero calcium is taken as unity). Background release in normal saline was subtracted from the release under the 2 conditions shown (high $\mathrm{K}^{+}$, zero added $\mathrm{Ca}^{2+}$, and high $\mathrm{K}^{+}, 5 \mathrm{mM} \mathrm{Ca}^{2+}$ ).

of the biological function of the APRPs, however, are not yet resolved. Circumstantial evidence for a hormonal role, however, is provided by a demonstration that the APRPs are released from the $\mathrm{CC}$ in a voltage- and calcium-dependent manner. Figure 7 shows that both APRP 1 and APRP 2 are released from the $\mathrm{CC}$ by exposure to saline containing $50 \mathrm{~mm}$ potassium. The above-background (release in normal saline subtracted) release is approximately 5 times greater in the presence of $5 \mathrm{~mm}$ calcium. Note also that the 2 APRPs are released in approximately the same ratios as found in the $\mathrm{CC}$. That is, about twice as much APRP 1 as $\Lambda$ PRP 2. Similar release data have been previously published for the AKHs and AKH-homologous peptides (O'Shea et al., 1984; Pannabecker and Orchard, 1987). Our own release experiments show that the AKHs are co-released with the APRPs (not shown).

\section{Discussion}

Our results provide evidence for the existence of 2 new peptides derived from the 2 precursors of the AKH peptides. That P1 and $\mathrm{P} 2$ are precursors of $\mathrm{AKH} \mathrm{I}$ and $\mathrm{AKH}$ II is based on 2 types of evidence. First, pulse-chase experiments show that tryptophan incorporated into $\mathrm{P} 1$ and $\mathrm{P} 2$ is recovered in $\mathrm{AKH} \mathrm{I}$ and AKH II after processing. Second, P1 and P2 cross-react with an anti-AKH serum. Together these observations strongly support the role of $\mathrm{P} 1$ and $\mathrm{P} 2$ as $\mathrm{AKH}$ precursors (Hekimi and O'Shea, 1987). The evidence that $\mathrm{P} 1$ and $\mathrm{P} 2$ are in addition the precursors of APRP 1 and APRP 2 is derived from a series of quantitiative pulse-chase experiments. In summary, the P1 and $\mathrm{P} 2$ precursors incorporate tyrosine, an amino acid absent from the AKHs. The incorporated tyrosine can be recovered in the 2 identified APRPs when translation, but not processing, is blocked. No other tyrosine-labeled compound besides P1 and P2 can account for the amount of label appearing in the APRPs following a pulse-chase experiment. Thus, P1 and P2, the AKH precursors, appear to be the only candidates for the precursors of the APRPs. The strategy summarized above for the discovery of novel products of hormone precursor processing is perhaps possible only with a highly homogenous cell population such as found in the glandular lobes of the $\mathrm{CC}$.

In addition to providing evidence that the APRPs are derived from the AKH precursors, we also show that they are major fragments of their parent polypeptides. In fact, our size estimates show that P1 and P2 can each accommodate only 1 APRP and 1 but not more than 2 AKH sequences. The estimated size of $\mathrm{P} 1$ and $\mathrm{P} 2$ (8.4 $\mathrm{kDa}$ ) can accommodate very little more. The issue of the APRP and AKH content of the precursors will be resolved unambiguously only by determination of the complete amino acid sequences of $P 1$ and P2. Sequencing work on the Ps and APRPs is in progress (S. Hekimi and M. O'Shea, unpublished observations) and results confirm the predictions from the pulse-chase experiments reported here.

A reverse-phase chromatogram of extracts of the glandular cells of the CC shows only 4 major OD peaks. Two of these are AKH I and AKH II. The other 2 major OD peaks are the APRPs. Our present results show that 2 minor OD peaks are associated with $\mathrm{P} 1$ and $\mathrm{P} 2$. The amplitudes of these peaks reflect the steadystate levels of these precursors. Since precursors are not terminal products but metabolic intermediates, P1 and P2 do not accumulate and their steady-state levels would be expected to be low. In contrast, the 2 AKHs and the 2 APRPs are accumulating stored products of biosynthesis and are thus present in large amounts and generate the major OD peaks of the $\mathrm{CC}$ extract.

Several important questions remain to be addressed following our identification of APRPs in the locust CC. What, for example, are their functions? We know from other systems that peptide precursors often contain sequences of more than one type of biologically active peptide. Perhaps $\mathrm{P} 1$ and $\mathrm{P} 2$ are not exceptional in this respect, and the APRPs are peptide hormones with as yet undetermined biological activity. At present, the APRPs remain putative hormones as they were identified by a strategy in which biological activity was not used as a guide for purification. The AKHs and APRPs are however co-released by elevated potassium saline. Thus, the APRPs might be released at the initiation of flight behavior, as are the AKHs, and they may also be involved in regulating physiological functions important in that behavior.

\section{References}

Adams, M. E., and M. O'Shea (1983) Peptide co-transmitter at a neuromuscular junction. Science 221: 286-289.

Eipper, B. A., R. E. Mains, and E. Herbert (1986) Peptides in the nervous system. Trends Neurosci. 10: 463-468.

Hekimi, S., and M. O'Shea (1987) Identification of precursors of the insect neuropeptide adipokinetic hormone. J. Neurosci. 7: 2773-2784.

Lynch, D. R., and S. H. Snyder (1986) Neuropeptides: Multiple molecular forms, metabolic pathways, and receptors. Annu. Rev. Biochem. 55: 773-799.

Mayeri, E., B. S. Rothman, P. H. Brownell, W. D. Branton, and L. Padgett (1985) Nonsynaptic characteristics of neurotransmission mediated by egg-laying hormone in the abdominal ganglion of Aplysia. J. Neurosci. 5: 2060-2077.

O'Shea, M., J. Witten, and M. Schaffer (1984) Isolation and characterization of two myoactive neuropeptides: Further evidence of an invertebrate peptide family. J. Neurosci. 2: 521-529. 
Pannabecker, T. and I. Orchard (1987) Regulation of adipokinetic hormone release from locust neuroendocrine tissue: Participation of calcium and cyclic AMP. Brain Res. 423: 13-22.

Scheller, R. II., J. F. Jackson, L. B. McAllister, B. S. Rothman, E. Mayeri, and R. Axel (1983) A single gene encodes multiple neuropeptides mediating a sterotyped behavior. Cell 35: 7-22.

Siegert, K. J., P. J. Morgan, and W. Mordue (1985) Primary structures of locust adipokinetic hormones II. Hoppe Seylers Z. Physiol. Chem. 365: 723-727.
Sigvardt, K. A., B. S. Rothman, R. O. Brown, and E. Mayer (1986) The bag cells of Aplysia as a multitransmitter system: Identification of alpha bag cell peptide as a second neurotransmitter. J. Neurosci. 6: 803-813.

Stone, J. V., W. Mordue, K. E. Bartley, and H. R. Morris (1976) Structure of locust adipokinetic hormone that regulates lipid utilisation during flight. Nature 263: 207-211. 